\title{
Convivencia e inteligencia emocional en niños en edad escolar
}

\author{
Carmen Martorell ${ }^{1}$, Remedios González ${ }^{1}$, Paloma Rasal ${ }^{1}$ y Roberto Estellés ${ }^{2}$ \\ ${ }^{1}$ Universidad de Valencia (España) \\ ${ }^{2}$ Colegio San José (España)
}

La violencia o agresividad entre escolares es un tema de actualidad de gran relevancia social debido, principalmente, a que la escuela es una de las instituciones, junto a la familia, en la que se forman los niños y adolescentes. Para abordar este tema es imprescindible conocer, entre otras cosas, qué rasgos caracterizan a los alumnos violentos o agresivos. En este trabajo, se estudia la relación entre variables tales como empatía, autocontrol e impulsividad, que forman parte del constructo denominado inteligencia emocional, con influencia en las conductas agresivas en el ambiente escolar. Los resultados son alentadores ya que permiten diseñar programas concretos de intervención en relación con las variables edad, el sexo y empatía en relación con la agresividad.

Palabras clave: Bullying, agresividad, inteligencia emocional.

Living together and emotional intelligence in school-age children. The aggressivity or bullying between school students is a very important subject nowadays, and it is a significant social issue as school is an institution, where alongside the family, children and youth develop themselves. To approach this subject it is necessary to know what are the characteristics of children and youth who display bullying behaviour. In this research, we study the relationship between emotional intelligence variables, like empathy, self control and impulsivity, and their influence on aggressive behaviour at school. The results show us that aggressive behaviour is related to age, sex and empathy.

Key words: Bullying, aggressive behaviour, emotional intelligence.

Correspondencia: Carmen Martorell Pallás. Departamento de Personalidad, Evaluación y Tratamiento Psicológico. Facultad de Psicología. Universidad de Valencia. Avd. Blasco Ibáñez 21, C.P. 46022. Valencia (España). E-mail: carmen.martorell@uv.es 
La violencia entre escolares es tan antigua como la escuela misma, sin embargo, las investigaciones llevadas a cabo por varios autores (Cerezo, 1996, 2001, 2006; Ortega y Mora-Merchán, 2000; Serrano e Iborra, 2005; Avilés y Monjas, 2005; Piñuel y Oñate, 2005), muestran un incremento paulatino de las incidencias violentas, situándose en un índice cercano al $40 \%$, lo que pone de manifiesto que el problema de la agresividad entre escolares afecta a un gran número de niños y adolescentes, afectando tanto a su rendimiento académico como a su desarrollo personal por lo que, la víctima de agresión, puede desarrollar graves síntomas de ansiedad, percibiendo el ambiente escolar como aversivo y amenazante (Cerezo, 2006; Piñuel y Oñate, 2005).

Para poder intervenir en esta problemática es necesario delimitar conceptos y conocer cuáles son los factores que están directamente relacionados con el patrón de conducta que queremos prevenir o modificar. La falta de unicidad de criterios dificulta la tarea, sin embargo, autores relevantes han intentado, no sólo definir, sino analizar el contenido de las definiciones (Hernández, Sarabia y Casares, 2002; Avilés y Monjas, 2005; Díaz-Aguado, 2006; Stassen, 2007).

En cuanto a la violencia la OMS la define como el uso deliberado de la fuerza física o del poder, ya sea en grado de amenaza o efectivo, contra uno mismo, otra persona o un grupo o comunidad, que cause o tenga muchas probabilidades de causar lesiones, muerte, daños psicológicos, trastornos del desarrollo o privaciones.

El chico/a violento/a muestra deficiencias en dos cualidades esenciales de la inteligencia emocional: autocontrol y empatía (Henley y Long, 1999; Hernández et al., 2002; Avilés y Monjas, 2005; Díaz-Aguado, 2006; Stassen, 2007). En concreto, Olweus (1991) señala que los niños y adolescentes agresivos son impulsivos, tienen la necesidad de dominar a sus compañeros y no muestran empatía por las víctimas de sus agresiones

Olweus (1983), en referencia al ámbito escolar, definió el maltrato entre escolares o bullying como una acción negativa e intencionada donde se estable una relación de poder produciendo efectos negativo en la victima. En otras palabras, el bullying, hace referencia a un comportamiento repetitivo de fustigación e intimidación que tiende a producir aislamiento y exclusión social de la víctima. En esta misma línea cabe destacar otros estudios (Hernández et al. (2002); Avilés y Monjas, 2005; Moreno et al., 2006; Díaz-Aguado, 2006; Stassen, 2007).

Siguiendo los planteamientos de Olweus (1991) quien destaca la importancia que la impulsividad y la empatía en el desarrollo de la conducta violenta o agresiva, se ha planteado este trabajo. En concreto, se centra en tres aspectos de la inteligencia emocional: empatía, impulsividad y el afán de aventura y su relación con la agresividad en el centro educativo (Extremera, y Fernandez, 2004; Petrides, Furham y Fredereckson, 2004). Para ello se realizan análisis diferenciales teniendo en cuenta la variable edad, tal como diversos autores (Olweus, 1993; Sourander, Helstelä, Helenius y Piha, 2000; Nausel et al., 2001; Díaz-Aguado 2006), y la variable sexo como señalan (Boulton y 
Underwood, 1992; Olweus, 1993; Ortega y Mora-Merchán, 2000; Avilés y Monjas, 2005), por la importancia que han demostrado tener.

El objetivo general del trabajo es el estudio de la relación entre las variables de empatía, impulsividad y afán de aventura y la conducta agresiva en sujetos en edad escolar a través de una técnica sociométrica e instrumentos de autoinforme.

\section{METODO}

\section{Participantes}

La muestra total, extraída de un colegio público de la ciudad de Valencia, está formada por 108 sujetos cuyas edades están comprendidas entre los 9 y los 15 años.

Respecto al sexo, de los 108 sujetos, 46 son chicos y 62 son chicas, lo que en términos de porcentaje representa el $42,2 \%$ y el $56,9 \%$ respectivamente.

En cuanto a la edad, el rango es amplio, las categorías de edad que cuentan con más sujetos son: la de 10 años con 13 personas, lo que supone un 19,3\% del total; la de 11 años con 9 sujetos y representa el 19,3\%; la de 12 años con 15 sujetos equivalente al 26,6\%; y por último, la de 16 años con 12 sujetos, que representa un $15,6 \%$ de la muestra total.

La media de edad de la muestra total es de 12 años, con una DT de 1,66. La media de edad de las chicas es de 12 con un DT de 1,7 y la media de edad de los chicos es de 11,5 con una DT de 1,5, no habiendo diferencias significativas entre las medias de los grupos.

\section{Instrumentos}

Los instrumentos utilizados en esta investigación han sido tres uno de ellos de tipo sociométrico y los otros dos instrumentos de autoinforme. En concreto son:

Bull-S, Este instrumento, de tipo sociométrico, ha sido elaborado por Cerezo (2000). Los diversos estudios realizados han demostrado que es útil para la detección, medida y valoración de situaciones de agresividad entre escolares, entendiendo por conductas agresivas los insultos, amenazas, rechazo, peleas, etc. Posee tres objetivos fundamentales: Facilitar el análisis de las características socio-afectivas del grupo de iguales; Ayudar al profesorado en la detección de situaciones de abuso entre escolares; y Avanzar en la elaboración de propuestas de intervención. Las puntuaciones obtenidas en este instrumento sirven de criterio para clasificar a los sujetos en tres grupos criteriales.

Cuestionario ASB. Este instrumento fue elaborado por Allsopp y Feldman (1974, 1975, 1976) y traducido y adaptado al español por Martorell y Silva (1993). A través de este cuestionario se evalúa la "conducta antisocial" en el sentido de "agresión socializada", es decir, conductas predelictivas de transgresión de normas sociales. 
IVE-J Escala de Impulsividad, Afán de Aventura y Empatía, este cuestionario elaborado por Eysenck, Easting y Pearson (1984) fue traducido y adaptado al español por Martorell y Silva (1993). Consta de un total de tres escalas: impulsividad, afán de aventura y empatía.

\section{Procedimiento}

Todos los instrumentos fueron aplicados por la misma evaluadora leyendo las instrucciones en cada uno de los casos. La aplicación fue realizada durante el horario escolar, en horas de tutoría y en grupos formados entre 20 y 30 sujetos.

\section{RESULTADOS}

Recuento de frecuencias y porcentajes. Los resultados obtenidos a través del BULL-S se muestran en la tabla 1, en la que figuran tres categorías establecidas en el instrumento (agresivos, semi-agresivos y no-agresivos), la frecuencia y el porcentaje de sujetos en cada una de ellas, constituyendo cada categoría un grupo criterial.

Tabla 1. Distribución de la muestra según los resultados obtenidos en el BULL-S. Grupos criteriales

\begin{tabular}{ccc}
\hline G. CRITERIALES & FRECUENCIA & PORCENTAJE \\
\hline Agresivos & 4 & 3,6 \\
\hline Semi. Agresivos & 35 & 31,8 \\
\hline No agresivos & 70 & 63,6 \\
\hline
\end{tabular}

Los no-agresivos son los sujetos que no obtuvieron ninguna nominación por parte de sus compañeros en la categoría de acosador o víctima. Los sujetos agresivos son los que obtuvieron al menos el $25 \%$ de las nominaciones del grupo. Los sujetos semi-agresivos son aquellos que sí habían sido nominados alguna vez.

Si bien, en términos absolutos es mayor la cantidad de sujetos que no muestran agresividad (65\%), sin embargo, no debe pasarse por alto que, la primera y segunda categoría representan un $36 \%$.

En la figura 1 se presenta la distribución de la muestra total por sexo, en ella se aprecia la diferencia que existe entre chicos y chicas, en términos de porcentajes en cada una de las categorías.

Estos resultados muestran que son las chicas las que realizan un porcentaje más elevado de conductas agresivas de acoso (3\%) aunque, si se tiene en cuenta la categoría de las conductas semi-agresivas, son los chicos los que alcanzan un porcentaje mayor (20\%). En conjunto y con una diferencia importante, son las chicas (43\%) las que no emiten ninguna conducta agresiva o de acoso. Por lo tanto, hay diferencias a nivel de porcentajes en cuanto a la información obtenida a través del BULL-S en la variable sexo. 
Figura 1. Distribución de frecuencias en las categorías del BULL-S en la variable sexo

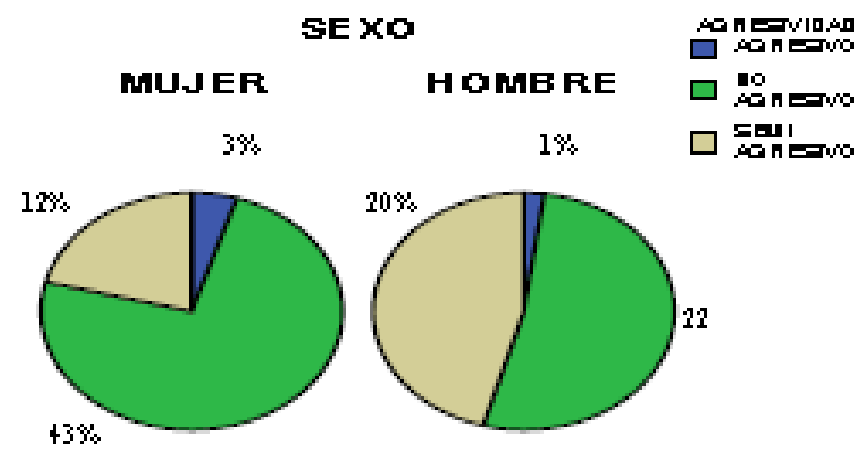

Cuando la variable moduladora es la edad, los resultados muestran cierta variabilidad. Cuando se toma como referencia los 10 años se observa un incremento de agresividad hacia los 11 años, disminuyendo entre los 12 y 13 años y volviéndose a incrementar a los 15 años.

Respecto a las conductas no agresivas, éstas, se incrementan claramente con la edad. En futuros trabajos hay que investigar sobre este punto para poder concluir si es que en realidad las conductas agresivas disminuyen con la edad o bien que las conductas que, en años inferiores eran consideradas como agresivas, con los años, cambia la percepción, la interpretación de las mismas o bien las conductas.

\section{Análisis correlacionales}

En primer lugar se ha procedido a realizar el análisis de correlaciones entre escalas en la muestra total. Los resultados se presentan en la tabla 2.

Tabla 2. Matriz de correlaciones en la muestra total entre el IVE-J, ASB y el BULL-S

\begin{tabular}{lcccc}
\hline & \multirow{2}{*}{ ASB } & \multirow{2}{*}{ BULL-S } & \multicolumn{3}{c}{ IVE-J } \\
\cline { 3 - 5 } & & Impulsi. & A. Aventura & Empatía \\
\hline ASB &,- 03 &, $48^{* *}$ &, 17 &, 01 \\
\hline BULL-S & &, 03 &, 07 & $-21^{*}$ \\
\hline IVE-J & &, $37^{* *}$ &, 05 \\
\hline Impulsi. & & &, 10 \\
\hline A. Aventura & & & - \\
\hline Empatía & & & \\
\hline
\end{tabular}

En el conjunto de las correlaciones, sólo hay tres índices con nivel de significación estadística. La escala de Impulsividad del IVE-J correlaciona positivamente con la escala de Afán de Aventura del mismo instrumento y con la escala de Conducta Antisocial (ASB). El BULL-S correlaciona negativamente con la escala de Empatía del IVE-J. En la información que aparece en la tabla 3, también puede comprobarse que las 
escalas ASB y la BULL-S no comparten nada de varianza, lo mismo ocurre en relación con las escalas de Impulsividad y Afán de Aventura del IVE-J.

Con la muestra total, tomando como variable moduladoras la edad y el sexo, se han realizado correlaciones con las puntuaciones obtenidas en cada uno de los instrumentos. En la tabla 3 se presenta el conjunto de las correlaciones.

Tabla 3. Matriz de correlaciones entre las variables sexo y edad en la muestra total con el IVE-J, ASB y el BULL-S

\begin{tabular}{lcc}
\hline ESCALAS & EDAD & SEXO \\
\hline ABS &, $40^{* *}$ &,- 07 \\
\hline BULL-S &,$- 33^{* *}$ &, $26 * *$ \\
\hline IVE-J & & \\
\hline Impulsividad &, $29 * *$ &, 10 \\
\hline Afán de Aventura &, 09 &, 08 \\
\hline Empatia &, $25 * *$ &,$- 35 * *$ \\
\hline
\end{tabular}

Estos resultados muestran que la variable edad correlaciona, con nivel de significación estadística, con todas las escalas, menos con la de Afán de Aventura, donde dicha variable se muestra independiente. Las escalas ASB, Impulsividad y Empatía lo hacen en sentido positivo; mientras que la agresividad evaluada a través del BULL-S correlaciona negativamente con la edad. Esta última, indica que la agresividad, evaluada por el ASB, es diferente a la evaluada por el BULL-S. En primer lugar, en la ASB se evalúan conductas consideradas previamente como transgresoras de la norma y en el BULL-S, se evalúan conductas de acoso; en segundo lugar, aunque hay que tener también en cuenta que un instrumento es de autoevaluación y el otro es sociométrico.

La tendencia no es la misma cuando se trata de la variable sexo, en la que sólo aparecen dos correlaciones con significación estadística, en concreto, el BULL-S correlaciona positivamente con el sexo, mientras que la escala de Empatía correlaciona negativamente.

\section{Análisis diferenciales}

En este apartado sólo se ha trabajado tomando como referencia la variable sexo, en cada una de las escalas, por ser más relevante en este estudio. Por las características de la muestra, para el estudio de las diferencias se ha recurrido a la $t$ de Student.

La variable moduladora ha sido las puntuaciones obtenidas en el BULL-S. Sin embargo, para estos análisis, los tres grupos establecidos en un principio se han reducido a dos: los agresivos/semi-agresivos y los no-agresivos, o sea, presencia de agresividad o acoso hacia los compañeros vs. ausencia de conductas agresivas o acoso hacia los compañeros. En la tabla 4 se presentan los resultados. 
Tabla 4. Diferencias entre grupos (agresivos vs no-agresivos BULL-S) a través de la ASB y del IVE-J en la muestra total

\begin{tabular}{|c|c|c|c|c|c|}
\hline ESCALAS & GRUPOS & $\mathrm{N}$ & Media & DT & Significación \\
\hline \multirow{2}{*}{ ABS } & Agresivo & 38 & 5,44 & 5,65 & \multirow{2}{*}{ NO } \\
\hline & No agresivo & 70 & 6,27 & 5,67 & \\
\hline \multirow{2}{*}{ IVE-J Impulsividad } & Agresivo & 38 & 9,50 & 4,45 & \multirow{2}{*}{ NO } \\
\hline & No agresivo & 70 & 9,24 & 3,71 & \\
\hline \multirow{2}{*}{$\begin{array}{l}\text { IVE-J Afán de } \\
\text { Aventura }\end{array}$} & Agresivo & 38 & 15,97 & 4,20 & \multirow{2}{*}{ NO } \\
\hline & No agresivo & 70 & 15,81 & 4,00 & \\
\hline \multirow{2}{*}{ IVE-J Empatía } & Agresivo & 38 & 16,36 & 2,91 & \multirow{2}{*}{ NO } \\
\hline & No agresivo & 70 & 17,52 & 3,37 & \\
\hline
\end{tabular}

Sólo comentar, que no hay diferencias en ninguna de las escalas cuando la variable moduladora son las categorías establecidas por el $B U L L-S$.

Tabla 5. Diferencias entre grupos (agresivos vs no-agresivos BULL-S) en la variable edad a través de la ASB y del IVE-J en la variable sexo

\begin{tabular}{|c|c|c|c|c|c|c|c|c|c|c|c|}
\hline \multicolumn{6}{|c|}{ MUJERES } & \multicolumn{6}{|c|}{ HOMBRES } \\
\hline ESCALAS & GRUPOS & $\mathrm{N}$ & Media & DT & Significación & ESCALAS & GRUPOS & $\mathrm{N}$ & Media & DT & Significación \\
\hline \multirow{2}{*}{ ABS } & Agresivo & 16 & 5,31 & 6,52 & \multirow{2}{*}{ NO } & \multirow{2}{*}{ ABS } & Agresivo & 20 & 5,10 & 4,20 & \multirow{2}{*}{ NO } \\
\hline & No agresivo & 44 & 6,95 & 5,99 & & & No agresivo & 24 & 5,20 & 5,17 & \\
\hline \multirow{2}{*}{$\begin{array}{l}\text { IVE-J } \\
\text { Impulsividad }\end{array}$} & Agresivo & 16 & 8,56 & 4,06 & \multirow{2}{*}{ NO } & \multirow{2}{*}{$\begin{array}{l}\text { IVE-J } \\
\text { Impulsividad }\end{array}$} & Agresivo & 20 & 10,30 & 4,74 & \multirow{2}{*}{ NO } \\
\hline & No agresivo & 44 & 9,00 & 3,68 & & & No agresivo & 24 & 9,37 & 3,94 & \\
\hline \multirow{2}{*}{$\begin{array}{l}\text { IVE-J Afán } \\
\text { de Aventura }\end{array}$} & Agresivo & 16 & 15,31 & 3,89 & \multirow{2}{*}{ NO } & \multirow{2}{*}{$\begin{array}{l}\text { IVE-J Afán } \\
\text { de Aventura }\end{array}$} & Agresivo & 20 & 16,7 & 4,54 & \multirow{2}{*}{ NO } \\
\hline & No agresivo & 44 & 15,81 & 4,09 & & & No agresivo & 24 & 15,75 & 4,01 & \\
\hline \multirow{2}{*}{$\begin{array}{l}\text { IVE-J } \\
\text { Empatía }\end{array}$} & Agresivo & 16 & 17,37 & 2,55 & \multirow{2}{*}{ NO } & IVE-J & Agresivo & 20 & 16,00 & 2,90 & \multirow{2}{*}{ NO } \\
\hline & No agresivo & 44 & 18,31 & 2,86 & & Empatía & No agresivo & 24 & 16,08 & 3,85 & \\
\hline
\end{tabular}

Tabla 6. Diferencias entre grupos (agresivos vs no-agresivos BULL-S) a través de la ASB y del IVE-J entre hombres y mujeres

\begin{tabular}{|c|c|c|c|c|c|c|c|c|c|c|c|}
\hline \multicolumn{6}{|c|}{ GRUPO AGRESIVO } & \multicolumn{6}{|c|}{ GRUPO NO AGRESIVO } \\
\hline ESCALAS & GRUPOS & $\mathrm{N}$ & Media & DT & Significación & ESCALAS & GRUPOS & $\mathrm{N}$ & Media & DT & Significación \\
\hline \multirow{2}{*}{ ABS } & MUJERES & 16 & 5,31 & & \multirow{2}{*}{ NO } & \multirow{2}{*}{ ABS } & MUJERES & 45 & 6,87 & 5,95 & \multirow{2}{*}{ NO } \\
\hline & HOMBRES & 22 & 5,55 & 5,07 & & & HOMBRES & 24 & 5,42 & 5,05 & \\
\hline IVE-J & MUJERES & 16 & 8,56 & 4,06 & \multirow{2}{*}{ NO } & IVE-J & MUJERES & 45 & 8,87 & 3,73 & \multirow{2}{*}{ NO } \\
\hline Impulsividad & HOMBRES & 22 & 10,18 & 4,69 & & Impulsividad & HOMBRES & 24 & 9,42 & 3,91 & \\
\hline \multirow{2}{*}{$\begin{array}{l}\text { IVE-J Afán } \\
\text { de Aventura }\end{array}$} & MUJERES & 16 & 15,31 & 3,89 & \multirow{2}{*}{ NO } & IVE-J Afán & MUJERES & 45 & 15,56 & 3,94 & \multirow{2}{*}{ NO } \\
\hline & HOMBRES & 22 & 16,45 & 4,43 & & de Aventura & HOMBRES & 24 & 16,08 & 4,10 & \\
\hline IVE-J & MUJERES & 16 & 17,38 & 2,55 & \multirow{2}{*}{0,060} & IVE-J & MUJERES & 45 & 18,29 & 2,88 & \multirow{2}{*}{0,005} \\
\hline Empatía & HOMBRES & 22 & 15,64 & 3,00 & & Empatía & HOMBRES & 24 & 15,96 & 3,73 & \\
\hline
\end{tabular}

Cuando la comparación se establece en un mismo sexo, teniendo como referencias los grupos criteriales establecidos, tal y como se observa en la tabla 5, tampoco hay diferencias significativas. Sin embargo, cuando la comparación se realiza, teniendo como referencia la variable sexo, dentro de cada una de las categorías (agresivos vs. No-agresivos) en cada una de las escalas, los resultados son los que se presentan en la tabla 6. 
En este caso aparece sólo una diferencia estadísticamente significativa, que indica, que tanto en el grupo agresivo, como en el grupo no-agresivo, las chicas puntúan más elevado en la escala de empatía (IVE-J) siendo mayor la diferencia en el grupo no-agresivo. Aunque no es muy grande, la variabilidad también ha de tenerse en cuenta, ya que aparece una tendencia a mostrarse más homogéneo el grupo de sujetos agresivos que el grupo de los sujetos no-agresivos.

\section{DISCUSION}

Las conclusiones más relevantes extraídas de los resultados obtenidos son:

- Hay un patrón de conducta distinto, según el sexo, en cuanto a la emisión de conductas relacionadas con la agresividad en el ambiente escolar tal y como demuestran los resultados obtenidos con el BULL-S. Hay más chicas que emiten conductas agresivas, sin embargo, cuando se trata de conductas semi-agresivas su número es bastante inferior al de los chicos. En la categoría de conductas no-agresivas, el grupo de mujeres es el más numeroso. En cuanto a la variable edad, hay un pico de conductas agresivas en torno a los 10-12 años, disminuyendo posteriormente la tendencia, apareciendo un incremento a los 15 años señalada por Olweus (1993) o Díaz-Aguado (2006) entre otros.

- Cuando se trata de conductas antisociales evaluadas con la escala $A S B$, la variable edad es más importante que la variable sexo. Los resultados ponen de manifiesto, que a más edad, se emiten más conductas antisociales, al tiempo que se es más impulsivo y más empático, sin embargo, la edad correlaciona inversamente con las conductas evaluadas con el BULL-S. Respecto al sexo, el número de correlaciones es menor.

- En cuanto al IVE-J ofrece información básica para formular programas de intervención. Los aspectos por él evaluados, están ligados con aspectos positivos que ayudan a la convivencia y que son incompatibles con la agresividad, razón por la cual, empatía correlaciona negativamente con la agresividad, evaluada a través de una prueba de tipo sociométrico, en la que la percepción del agredido determina el impacto psicológico de la agresión. En cambio, la empatía no correlaciona con las conductas antisociales evaluadas a través del $A S B$, que evalúa conductas que el sujeto realiza sin necesidad de implicar a otro.

- Respecto a las diferencias entre los grupos criteriales establecidos a través del BULL-S, no discriminan cuando se trata de conductas antisociales o contra la norma $(A S B)$, tampoco discriminan cuando se evalúa con el IVE-J la escala de impulsividad, aspecto importante de la extraversión, y la de afán de aventura, sin embargo, cuando se trata de la escala de empatía, aparecen diferencias, ya que las chicas puntúan más elevado en los dos grupos criteriales 
Estos resultados ponen de manifiesto la importancia de la empatía como elemento favorecedor de la convivencia escolar, al tiempo que destacan la importancia que los instrumentos de evaluación tienen en la planificación y éxito de los programas de prevención o intervención (Martorell, 2005, 2006; Martorell, en prensa).

\section{REFERENCIAS}

Allsopp, J.F. (1975). Investigations into the applicability of Eysenck's theory criminality to the antisocial of schoolchildren. Doctoral Dissertation. University of London.

Allsopp, J.F. \& Feldman, M.P. (1974). Extraversion, neuroticism, psychoticism and antisocial behavior in schoolgirls. Social Behavior and Personality, 2, 184-190.

Allsopp, J.F. \& Feldman, M.P. (1976). The analysis of quetionnaire neasures of personality and antisocial behavior in schoolboys. British Journal of Criminology, 16, 337-351.

Avilés, J.M. y Monjas, I. (2005). Estudio de incidencia de la intimidación y el maltrato entre iguales en la educación secundaria obligatoria mediante el cuestionario CIME. Anales de Psicología, 21(1), 27-41.

Boulton, M.J. \& Underwood, K. (1992). Bully/victim problems among middle school children. British Journal of Educational Studies, 28(4), 353-370.

Cerezo, F. (1996). Agresividad social entre escolares. La dinámica bullying. Murcia: Servicio de publicaciones de la universidad de Murcia.

Cerezo, F. (2001). La violencia en las aulas. Madrid: Pirámide.

Cerezo, F. (2006). Violencia y victimización entre escolares. El bullying: estrategias de identificación y elementos para la intervención a través del Test Bull-S. Revista Electrónica de Investigación Psicoeducativa, 9(4), 333-352.

Díaz-Aguado, M.J. (2006). Sexismo, violencia de género y acoso escolar. Propuestas para una prevención integral de la violencia. Estudios de Juventud, 73, 38-58.

Eysenck, S.B.G., Easting, G. \& Pearson, P.R. (1984). Age norms for impulsiveness, venturesomeness and empathy in children. Personality and Individual Differences, 5, 315321.

Extremera, N. y Fernández, P. (2004). La inteligencia emocional. Métodos de evaluación en el aula. Revista Iberoamericana de Educación, 30, 1-12.

Extremera, N. y Fernández, P. (2004). El papel de la inteligencia emocional en el alumnado: evidencias empíricas. Revista Electrónica de Investigación Educativa, 6(2), 1-17.

Henley, M. \& Long, N.J. (1999). Teaching emocional intelligence to impulsive-aggressive youth. Reclaiming Children and Youth, 7(4), 224-229.

Hernández, T., Sarabia, B. y Casares, E. (2002). Incidencia de variables contextuales en la violencia "bullying" en el recinto escolar. Psicothema, 14(supl.), 50-62.

Martorell, M.C. (2005). Guía para la convivencia escolar (primaria). Valencia: Consellería de Educación.

Martorell, M.C. (2005). Guía para la convivencia escolar (secundaria). Valencia: Consellería de Educación.

Martorell, M.C. (en prensa). Casos y Soluciones: convivencia escolar. Valencia: Consellería de Educación. 
Martorell, M.C. y Silva, F. (1993). Escala de Impulsividad, Afán de Aventura y Empatía. En F. Silva y M.C. Martorell (Eds.), Evaluación de la personalidad infantil y juvenil, Madrid: Mepsa.

Martorell, M.C. y Silva, F. (1993). Escala de conducta antisocial ASB. En F. Silva y M.C. Martorell (Eds.), Evaluación de la personalidad infantil y juvenil, Madrid: Mepsa.

Nansel, T.R., Overpeck, M., Pilla, R.S., Ruan, W.J., Simons-Morton, B. \& Scheidt, P. (2001). Bullying behaviours among US youth: prevalence and association with psychosocial adjustment. Journal of the American Medical Association, 285(16), 2094-2100.

Olweus, D. (1993). Conductas de acoso y amenaza entre escolares. Madrid: Morata.

Organización Mundial de la Salud. OMS. (2003). La violencia, un problema mundial de salud pública. http://www.who.int/about/es/

Ortega, R. y Mora-Merchán, J.A. (2000). Violencia escolar. Mito o realidad. Sevilla: Mergablum.

Petrides, K.V., Frederickson, N. \& Furnham, A. (2004). The role of trait emotional intelligence in academic performance and deviant behaviour at school. Personality and Individual Differences, 36(2), 277-293.

Piñuel, I. y Oñate, A. (2005). Informe Cisneros VII: violencia y acoso escolar en alumnos de primaria, ESO y Bachiller (Informe preliminar). Instituto de Innovación Educativa y Desarrollo Directivo. (http://www.acosoescolar.com)

Serrano, A. y Iborra, I. (2005). Informe: Violencia entre compañeros en la escuela. Centro Reina Sofía para el estudio de la violencia. (http://www.gva.es/violencia)

Sourander, A., Helstelä, L., Helenius, H. \& Piha, J. (2000). Persistence of bullying from childhood to adolescence: a longitudinal 8-years follow-up study. Child abuse \& Neglect, 24(7), 873881.

Stassen, K. (2007). Update on bullying at school: science forgotten? Developmental Review, 27, 90-126.

Recibido: 21 de julio de 2008

Modificaciones: 31 de noviembre de 2008

Aceptado: 11 de diciembre de 2008 